\title{
A parameter-dependent refinement of the discrete Jensen's inequality for convex and mid-convex functions
}

László Horváth

Correspondence: Ihorvath@almos. vein.hu

Department of Mathematics, University of Pannonia, 8200 Veszprém, Egyetem u. 10., Hungary

\begin{abstract}
In this paper, a new parameter-dependent refinement of the discrete Jensen's inequality is given for convex and mid-convex functions. The convergence of the introduced sequences is also studied. One of the proofs requires an interesting convergence theorem with probability theoretical background. We apply the results to define some new quasi-arithmetic and mixed symmetric means and study their monotonicity and convergence.
\end{abstract}

\section{Introduction and the main results}

The considerations of this paper concern

$\left(\mathrm{A}_{1}\right)$ an arbitrarily given real vector space $X$, a convex subset $C$ of $X$, and a finite subset $\left\{x_{1}, \ldots, x_{n}\right\}$ of $C$, where $n \geq 1$ is fixed;

$\left(\mathrm{A}_{2}\right)$ a convex function $f: C \rightarrow \mathbb{R}$, and a discrete distribution $p_{1}, \ldots, p_{n}$, which means that $p_{j} \geq 0$ with $\sum_{j=1}^{n} p_{j}=1$;

$\left(\mathrm{A}_{3}\right)$ a mid-convex function $f: C \rightarrow \mathbb{R}$, and a discrete distribution $p_{1}, \ldots, p_{n}$ with rational $p_{j}(1 \leq j \leq n)$.

The function $f: C \rightarrow \mathbb{R}$ is called convex if

$$
f(\alpha x+(1-\alpha) y) \leq \alpha f(x)+(1-\alpha) f(y), \quad x, y \in C, \quad 0 \leq \alpha \leq 1,
$$

and mid-convex if

$$
f\left(\frac{x+y}{2}\right) \leq \frac{1}{2} f(x)+\frac{1}{2} f(y), \quad x, y \in C .
$$

For a variety of applications, the discrete Jensen's inequalities are important:

Theorem A. (see [1]) (a) If $\left(A_{1}\right)$ and $\left(A_{2}\right)$ are satisfied, then

$$
f\left(\sum_{j=1}^{n} p_{j} x_{j}\right) \leq \sum_{j=1}^{n} p_{j} f\left(x_{j}\right) .
$$

(b) If $\left(A_{1}\right)$ and $\left(A_{3}\right)$ are satisfied, then (2) also holds.

Let $\mathbb{N}:=\{0,1,2, \ldots\}$ and let $\mathbb{N}_{+}:=\{1,2, \ldots\}$. 
Various attempts have been made to refine inequality (2) in the following ways: Assume either $\left(\mathrm{A}_{1}\right)$ and $\left(\mathrm{A}_{2}\right)$ or $\left(\mathrm{A}_{1}\right)$ and $\left(\mathrm{A}_{3}\right)$. Let $m \geq 2$ be an integer, and let $I$ denote either the set $\{1, \ldots, m\}$ or the set $\mathbb{N}_{+}$.

(B) Create a decreasing real sequence $\left(B_{k}\right)_{k \in I}$ such that $B_{k}=B_{k}\left(f, x_{i}, p_{i}\right)(k \in I)$ is a sum whose index set is a subset of $\{1, \ldots, n\}^{k}$ and

$$
f\left(\sum_{j=1}^{n} p_{j} x_{j}\right) \leq \cdots \leq B_{k} \leq \cdots \leq B_{1}=\sum_{j=1}^{n} p_{j} f\left(x_{j}\right), \quad k \in I .
$$

(C) Create an increasing real sequence $\left(C_{k}\right)_{k \in I}$ such that $C_{k}=C_{k}\left(f, x_{i}, p_{i}\right)(k \in I)$ is a sum whose index set is a subset of $\{1, \ldots, k\}^{n}$ and

$$
f\left(\sum_{j=1}^{n} p_{j} x_{j}\right)=C_{1} \leq \cdots \leq C_{k} \leq \cdots \leq \sum_{j=1}^{n} p_{j} f\left(x_{j}\right), \quad k \in I .
$$

The next two typical results belong to the group of refinements of type (B).

These examples use $p_{1}=\cdots=p_{n}=\frac{1}{n}$. In [2], Pečarić and Volenec have constructed the sequence

$$
f_{k}:=\frac{1}{\left(\begin{array}{l}
n \\
k
\end{array}\right)} \sum_{1 \leq i_{1}<\cdots<i_{k} \leq n} f\left(\frac{x_{i_{1}}+\cdots+x_{i_{k}}}{k}\right), \quad 1 \leq k \leq n,
$$

while the other sequence

$$
\bar{f}_{k}:=\frac{1}{\left(\begin{array}{c}
n+k-1 \\
k
\end{array}\right)} \sum_{1 \leq i_{1} \leq \cdots \leq i_{k} \leq n} f\left(\frac{x_{i_{1}}+\cdots+x_{i_{k}}}{k}\right), \quad k \in \mathbb{N}_{+}
$$

is due to Pečarić and Svrtan [3]. In a recent work, [4] Horváth and Pečarić define a lot of new sequences, they generalize and give a uniform treatment a number of wellknown results from this area, especially (5) and (6) are extended. Horváth develops a method in [5] to construct decreasing real sequences satisfying (3). His paper contains some improvements of the results in [4] and gives a new approach of the topic. The description of the sequences in $[4,5]$ requires some work, so we do not go into the details. The problem (B) has been considered for the classical Jensen's inequality by Horváth [6].

We turn now to the group of refinements of type (C). In contrast to the previous problem, it is not easy to find such results. Recently, Xiao et al. [7] have obtained the sequence

$$
F_{k}:=\frac{1}{\left(\begin{array}{c}
n+k-2) \\
k-1
\end{array}\right)} \sum_{\substack{i_{1}+\cdots+i_{n}=n+k-1 \\
i_{j} \in \mathbb{N}_{+}(1 \leq j \leq n)}} f\left(\frac{1}{n+k-1} \sum_{j=1}^{n} i_{j} x_{j}\right), \quad k \in \mathbb{N}_{+}
$$

which satisfies (4) with $p_{1}=\cdots=p_{n}=\frac{1}{n}$.

In this paper, we establish a new solution of the problem $(C)$. The constructed sequence $\left(C_{k}(\lambda)\right)_{k \geq 0}$ depends on a parameter $\lambda$ belonging to [1, $\infty$, and we can use arbitrary discrete distribution $p_{1}, \ldots, p_{n}$, not just the appropriate discrete uniform distribution. We give the limit of the sequence under fixed parameter. We also study the 
convergence of the sequence when the parameter varies and $k \in \mathbb{N}$ is fixed. Finally, some applications are given which concern the theme of means.

The next theorems are the main results of this paper. We need some further hypotheses:

$\left(\mathrm{A}_{4}\right)$ Let $\lambda \geq 1$.

$\left(\mathrm{A}_{5}\right)$ Let $\lambda \geq 1$ be rational.

First, we give a refinement of the discrete Jensen's inequality (2).

Theorem 1 Suppose either $\left(A_{1}\right),\left(A_{2}\right)$, and $\left(A_{4}\right)$ or $\left(A_{1}\right),\left(A_{3}\right)$, and $\left(A_{5}\right)$. Introduce the sets

$$
S_{k}:=\left\{\left(i_{1}, \ldots, i_{n}\right) \in \mathbb{N}^{n} \mid \sum_{j=1}^{n} i_{j}=k\right\}, \quad k \in \mathbb{N},
$$

and for $k \in \mathbb{N}$ define the numbers

$$
\begin{gathered}
C_{k}(\lambda)=C_{k}\left(x_{1}, \ldots, x_{n} ; p_{1}, \ldots, p_{n} ; \lambda\right) \\
:=\frac{1}{(n+\lambda-1)^{k}} \sum_{\left(i_{1}, \ldots, i_{n}\right) \in S_{k}} \frac{k !}{i_{1} ! \ldots i_{n} !}\left(\sum_{j=1}^{n} \lambda^{i_{j}} p_{j}\right) f\left(\frac{\sum_{j=1}^{n} \lambda^{i_{j}} p_{j} x_{j}}{\sum_{j=1}^{n} \lambda^{i_{j}} p_{j}}\right) .
\end{gathered}
$$

Then,

$$
f\left(\sum_{j=1}^{n} p_{j} x_{j}\right)=C_{0}(\lambda) \leq C_{1}(\lambda) \leq \cdots \leq C_{k}(\lambda) \leq \cdots \leq \sum_{j=1}^{n} p_{j} f\left(x_{j}\right), \quad k \in \mathbb{N} .
$$

Remark 2 (a) It follows from the definition of $S_{k}$ that $S_{k} \subset\{0, \ldots, k\}^{n}(k \in \mathbb{N})$.

(b) It is easy to see that

$$
C_{k}(1)=f\left(\sum_{j=1}^{n} p_{j} x_{j}\right), \quad k \in \mathbb{N} .
$$

Finally, we establish two convergence theorems.

Theorem 3 Suppose $\left(A_{1}\right),\left(A_{2}\right)$, and $\left(A_{4}\right)$. Suppose $\times$ is a normed space and $f$ is continuous. Then,

(a) For every fixed $\lambda>1$

$$
\lim _{k \rightarrow \infty} C_{k}(\lambda)=\sum_{j=1}^{n} p_{j} f\left(x_{j}\right)
$$

(b) The function $\lambda \rightarrow C_{k}(\lambda)(\lambda \geq 1)$ is continuous for every $k \in \mathbb{N}$.

The proof of Theorem 3(a) requires a lemma (see Lemma 15), which is interesting in its own right. Probability theoretical technique will be used to handle this problem. 
Remark 4 In the previous theorem, it suffices to consider the case when $\left(A_{1}\right),\left(A_{2}\right)$, and $\left(A_{4}\right)$ are satisfied. Really, if $f$ is mid-convex and continuous, then convex.

By (9)

$$
\lim _{k \rightarrow \infty} C_{k}(1)=f\left(\sum_{j=1}^{n} p_{j} x_{j}\right) .
$$

We come now to the second convergence theorem.

Theorem 5 Suppose either $\left(A_{1}\right),\left(A_{2}\right)$, and $\left(A_{4}\right)$ or $\left(A_{1}\right),\left(A_{3}\right)$, and $\left(A_{5}\right)$. For each fixed $k \in \mathbb{N}_{+}$

$$
\lim _{\lambda \rightarrow \infty} C_{k}(\lambda)=\sum_{j=1}^{n} p_{j} f\left(x_{j}\right) .
$$

\section{Discussion and applications}

Suppose either $\left(A_{1}\right),\left(A_{2}\right)$, and $\left(A_{4}\right)$ or $\left(A_{1}\right),\left(A_{3}\right)$, and $\left(A_{5}\right)$. First, we give three special cases of (8).

(a) $k=1, n \in \mathbb{N}_{+}$:

$$
C_{1}(\lambda)=\frac{1}{n+\lambda-1} \sum_{i=1}^{n}\left(1+(\lambda-1) p_{i}\right) f\left(\frac{\sum_{j=1}^{n} p_{j} x_{j}+(\lambda-1) p_{i} x_{i}}{1+(\lambda-1) p_{i}}\right) .
$$

(b) $k \in \mathbb{N}, n=2$ :

$$
C_{k}(\lambda)=\frac{1}{(\lambda+1)^{k}} \sum_{i=0}^{k}\left(\begin{array}{c}
k \\
i
\end{array}\right)\left(\lambda^{i} p_{1}+\lambda^{k-i} p_{2}\right) f\left(\frac{\lambda^{i} p_{1} x_{1}+\lambda^{k-i} p_{2} x_{2}}{\lambda^{i} p_{1}+\lambda^{k-i} p_{2}}\right) .
$$

(c) $p_{1}=\cdots=p_{n}:=\frac{1}{n}:$

$$
C_{k}(\lambda)=\frac{1}{n(n+\lambda-1)^{k}} \sum_{\left(i_{1}, \ldots, i_{n}\right) \in S_{k}} \frac{k !}{i_{1} ! \ldots i_{n} !}\left(\sum_{j=1}^{n} \lambda^{i_{j}}\right) f\left(\frac{\sum_{j=1}^{n} \lambda^{i_{j}} x_{j}}{\sum_{j=1}^{n} \lambda^{i_{j}}}\right) .
$$

Assume further that $f$ is strictly convex (strictly mid-convex) that is strict inequality holds in (1) whenever $x \neq y$ and $0<\alpha<1$. In this case, equality is satisfied in (2) if and only if $x_{1}=\cdots=x_{n}$, and therefore, it comes from the third part of the proof of Theorem 1 that

$$
C_{k}(\lambda)<\sum_{j=1}^{n} p_{j} f\left(x_{j}\right), \quad k \in \mathbb{N},
$$


if not all $x_{i}$ are equal.

If $p_{1}=\cdots=p_{n}:=\frac{1}{n}$ and $f$ is strictly convex (strictly mid-convex), then the analysis of the proof of Theorem 1 shows that

$$
f\left(\frac{1}{n} \sum_{j=1}^{n} x_{j}\right)=C_{0}(\lambda)<C_{1}(\lambda)<\cdots<C_{k}(\lambda)<\cdots<\frac{1}{n} \sum_{j=1}^{n} f\left(x_{j}\right), \quad k \in \mathbb{N},
$$

whenever not all $x_{i}$ are equal.

If the inequality (10) holds, $X$ is a normed space and $f$ is continuous (see Remark 4), then Theorem 3(b) and Theorem 5 insure that the range of the function $\lambda \rightarrow C_{k}(\lambda)$ $\left(k \in \mathbb{N}_{+}\right)$is the interval

$$
\left[f\left(\sum_{j=1}^{n} p_{j} x_{j}\right), \quad \sum_{j=1}^{n} p_{j} f\left(x_{j}\right)[.\right.
$$

Conjecture 6 Suppose either $\left(A_{1}\right),\left(A_{2}\right)$, and $\left(A_{4}\right)$ or $\left(A_{1}\right),\left(A_{3}\right)$, and $\left(A_{5}\right)$.

The function $\lambda \rightarrow C_{k}(\lambda)(\lambda \geq 1)$ is increasing for every $k \in \mathbb{N}$.

Next, we define some new quasi-arithmetic means and study their monotonicity and convergence. About means see [8].

Definition 7 Let $I \subset \mathbb{R}$ be an interval, let $x_{j} \in I(1 \leq j \leq n)$, let $p_{1}, \ldots, p_{n}$ be a discrete distribution, and let $g, h: I \rightarrow \mathbb{R}$ be continuous and strictly monotone functions. Let $\lambda \geq 1$. We define the quasi-arithmetic means with respect to (8) by

$$
\begin{aligned}
M_{h, g}(k, \lambda):= & h^{-1}\left(\frac{1}{(n+\lambda-1)^{k}} \sum_{\left(i_{1}, \ldots, i_{n}\right) \in S_{k}} \frac{k !}{i_{1} ! \ldots i_{n} !}\left(\sum_{j=1}^{n} \lambda^{i_{j}} p_{j}\right)\right. \\
& \left.\cdot\left(h \circ g^{-1}\right)\left(\frac{\sum_{j=1}^{n} \lambda^{i_{j}} p_{j} g\left(x_{j}\right)}{\sum_{j=1}^{n} \lambda^{i_{j}} p_{j}}\right)\right), \quad k \in \mathbb{N} .
\end{aligned}
$$

Some other means are also needed.

Definition 8 Let $I \subset \mathbb{R}$ be an interval, let $x_{j} \in I(1 \leq j \leq n)$, and let $p_{1}, \ldots, p_{n}$ be a discrete distribution. For a continuous and strictly monotone function $z: I \rightarrow \mathbb{R}$, we introduce the following mean

$$
M_{z}:=z^{-1}\left(\sum_{j=1}^{n} p_{j} z\left(x_{j}\right)\right)
$$

We now prove the monotonicity of the means (11) and give limit formulas.

Proposition 9 Let $I \subset \mathbb{R}$ be an interval, let $x_{j} \in I(1 \leq j \leq n)$, let $p_{1}, \ldots$, $p_{n}$ be a discrete distribution, and let $g, h: I \rightarrow \mathbb{R}$ be continuous and strictly monotone functions. Let $\lambda \geq 1$. Then,

(a)

$$
M_{g}=M_{h, g}(0, \lambda) \leq \cdots \leq M_{h, g}(k, \lambda) \leq \cdots \leq M_{h}, \quad k \in \mathbb{N},
$$


if either $h \circ g^{-1}$ is convex and $h$ is increasing or $h \circ g^{-1}$ is concave and $h$ is decreasing.

(b)

$$
M_{g}=M_{h, g}(0, \lambda) \geq \cdots \geq M_{h, g}(k, \lambda) \geq \cdots \geq M_{h}, \quad k \in \mathbb{N},
$$

if either $h \circ g^{-1}$ is convex and $h$ is decreasing or $h \circ g^{-1}$ is concave and $h$ is increasing.

(c) Moreover, in both cases

$$
\lim _{k \rightarrow \infty} M_{h, g}(k, \lambda)=M_{h}
$$

for each fixed $\lambda>1$, and

$$
\lim _{\lambda \rightarrow \infty} M_{h, g}(k, \lambda)=M_{h}
$$

for each fixed $k \in \mathbb{N}_{+}$.

Proof. Theorem 1 can be applied to the function $h \circ g^{-1}$, if it is convex $\left(-h \circ g^{1}\right.$, if it is concave) and the $n$-tuples $\left(g\left(x_{1}\right), \ldots, g\left(x_{n}\right)\right)$, then upon taking $h^{-1}$, we get (a) and (b). (c) comes from Theorems $3(\mathrm{a})$ and 5.

As a special case, we consider the following example.

Example 10 If $I:=] 0, \infty[, h:=\ln$, and $g(x):=x(x \in] 0, \infty[)$, then by Proposition $9(b)$, we have the following inequality. for every $x_{j}>0(1 \leq j \leq n), \lambda \geq 1$, and $k \in \mathbb{N}_{+}$

$$
\sum_{j=1}^{n} p_{j} x_{j} \geq \prod_{\left(i_{1}, \ldots, i_{n}\right) \in S_{k}}\left(\frac{\sum_{j=1}^{n} \lambda^{i_{j}} p_{j} x_{j}}{\sum_{j=1}^{n} \lambda^{i_{j}} p_{j}}\right)^{\frac{1}{(n+\lambda-1)^{k}} \frac{k !}{i_{1} ! \ldots i_{n} !} \sum_{j=1}^{n} \lambda^{i_{j}} p_{j}} \geq \prod_{j=1}^{n} x_{j}^{p_{j}},
$$

which gives a sharpened version of the arithmetic mean - geometric mean inequality

$$
\frac{1}{n} \sum_{j=1}^{n} x_{j} \geq \prod_{\left(i_{1}, \ldots, i_{n}\right) \in S_{k}}\left(\frac{\sum_{j=1}^{n} \lambda^{i_{j}} x_{j}}{\sum_{j=1}^{n} \lambda^{i_{j}}}\right)^{\frac{1}{n(n+\lambda-1)^{k}} \frac{k !}{i_{1} ! \ldots i_{n} !} \sum_{j=1}^{n} \lambda^{i_{j}}} \geq \prod_{j=1}^{n} x_{j}^{\frac{1}{n}} .
$$

Finally, we investigate some mixed symmetric means.

The power means of order $r \in \mathbb{R}$ are defined as follows:

Definition 11 Let $\left.x_{j} \in\right] 0, \infty\left[(1 \leq j \leq n)\right.$, and let $p_{1}, \ldots, p_{n}$ be a discrete distribution with $p_{j}>0(1 \leq j \leq n)$.

$$
M_{r}=M_{r}\left(x_{1}, \ldots, x_{n} ; p_{1}, \ldots, p_{n}\right):=\left\{\begin{array}{ll}
\left(\sum_{j=1}^{n} p_{j} x_{j}^{r}\right)^{\frac{1}{r}}, r \neq 0 \\
\left(\prod_{j=1}^{n} x_{j}^{p_{j}}\right)^{,}, r \neq 0
\end{array} .\right.
$$

If $r \neq 0$, then the power means of order $r$ belong to the means (12) $(z:] 0, \infty[\rightarrow \mathbb{R}, z$ $\left.(x):=x^{r}\right)$, while we get the power means of order 0 by taking limit. Supported by the power means, we can introduce mixed symmetric means corresponding to (8): 
Definition 12 Let $\left.x_{j} \in\right] 0, \infty\left[(1 \leq j \leq n)\right.$, and let $p_{1}, \ldots, p_{n}$ be a discrete distribution with $p_{j}>0(1 \leq j \leq n)$. Let $\lambda \geq 1$, and $k \in \mathbb{N}$. We define the mixed symmetric means with respect to (8) by

$$
\begin{gathered}
:=\left(\frac{1}{(n+\lambda-1)^{k}} \sum_{\left(i_{1}, \ldots, i_{n}\right) \in S_{k}} \frac{k !}{i_{1} ! \ldots i_{n} !}\left(\sum_{j=1}^{n} \lambda^{i_{j}} p_{j}\right)\right. \\
\left.\cdot M_{t}^{s}\left(x_{1}, \ldots, x_{n} ; \frac{\lambda^{i_{1}} p_{1}}{\sum_{j=1}^{n} \lambda^{i_{j}} p_{j}}, \ldots, \frac{\lambda^{i_{n}} p_{n}}{\sum_{j=1}^{n} \lambda^{i_{j}} p_{j}}\right)\right)^{\frac{1}{s}},
\end{gathered}
$$

if $s, t \in \mathbb{R}$ and $s \neq 0$, and

$$
\begin{gathered}
M_{0, t}(k, \lambda):=\prod_{\left(i_{1}, \ldots, i_{n}\right) \in S_{k}} \\
\left(M_{t}\left(x_{1}, \ldots, x_{n} ; \frac{\lambda^{i_{1}} p_{1}}{\sum_{j=1}^{n} \lambda^{i_{j}} p_{j}}, \ldots, \frac{\lambda^{i_{n}} p_{n}}{\sum_{j=1}^{n} \lambda^{i_{j}} p_{j}}\right)\right) \\
\frac{k !}{(n+\lambda-1)^{k}} \frac{i_{1} ! \ldots i_{n} !}{\left.i_{j=1}^{n} \lambda^{i_{j}} p j\right)}
\end{gathered}
$$

where $t \in \mathbb{R}$.

The monotonicity and the convergence of the previous means are studied in the next result.

Proposition 13 Let $\left.x_{j} \in\right] 0, \infty\left[(1 \leq j \leq n)\right.$, and let $p_{1}, \ldots, p_{n}$ be a discrete distribution with $p_{j}>0(1 \leq j \leq n)$. Let $\lambda \geq 1$, and $k \in \mathbb{N}$. Suppose $s, t \in \mathbb{R}$ such that $s \leq t$. Then,

(a)

$$
M_{t}=M_{s, t}(0, \lambda) \geq \cdots \geq M_{s, t}(k, \lambda) \geq \cdots \geq M_{s,} \quad k \in \mathbb{N} .
$$

(b) In case of $s, t \neq 0$

$$
\lim _{k \rightarrow \infty} M_{s, t}(k, \lambda)=M_{s}
$$

for each fixed $\lambda>1$, and

$$
\lim _{\lambda \rightarrow \infty} M_{s, t}(k, \lambda)=M_{s}
$$

for each fixed $k \in \mathbb{N}_{+}$.

Proof. Assume $s, t \neq 0$. Then, Proposition 9 (b) can be applied with $g, h:] 0, \infty[\rightarrow \mathbb{R}$, $g(x):=x^{t}$, and $h(x):=x^{s}$. If $s=0$ or $t=0$, the result follows by taking limit.

\section{Some lemmas and the proofs of the main results}

Lemma 14 Let $k \in \mathbb{N}$ and $\left(i_{1}, \ldots, i_{n}\right) \in S_{k+1}$ be fixed. If we set

$$
z\left(i_{1}, \ldots, i_{n}\right):=\left\{j \in\{1, \ldots, n\} \mid i_{j} \neq 0\right\},
$$


then

$$
\sum_{j \in z\left(i_{1}, \ldots, i_{n}\right)} \frac{k !}{i_{1} ! \ldots i_{j-1} !\left(i_{j}-1\right) ! i_{j+1} ! \ldots i_{n} !}=\frac{(k+1) !}{i_{1} ! \ldots i_{n} !} .
$$

Proof. The lowest common denominator is $i_{1} ! \ldots i_{n} !$. Combined with $\sum_{j=1}^{n} i_{j}=k+1$, the result follows.

The proof of Theorem 1 .

Proof. (a) We separate the proof of this part of the theorem into three steps.

Let $\lambda \geq 1$ be fixed.

I. Since $S_{0}=\{(0, \ldots, 0)\}$

$$
C_{0}(\lambda)=\left(\sum_{j=1}^{n} \lambda^{0} p_{j}\right) f\left(\frac{\sum_{j=1}^{n} \lambda^{0} p_{j} x_{j}}{\sum_{j=1}^{n} \lambda^{0} p_{j}}\right)=f\left(\sum_{j=1}^{n} p_{j} x_{j}\right) .
$$

II. Next, we prove that $C_{k}(\lambda) \leq C_{k+1}(\lambda)(k \in \mathbb{N})$.

It is easy to check that for every $\left(i_{1}, \ldots, i_{n}\right) \in S_{k}$

$$
\begin{gathered}
\frac{\sum_{j=1}^{n} \lambda^{i_{j}} p_{j} x_{j}}{\sum_{j=1}^{n} \lambda^{i_{j}} p_{j}}=\frac{1}{n+\lambda-1} \\
\cdot \sum_{l=1}^{n}\left(\frac{\sum_{j=1}^{n} \lambda^{i^{j}} p_{j} x_{j}+(\lambda-1) \lambda^{i_{l}} p_{l} x_{l}}{\sum_{j=1}^{n} \lambda^{i_{j}} p_{j}+(\lambda-1) \lambda^{{ }^{i}} p_{l}} \cdot \frac{\sum_{j=1}^{n} \lambda^{i} p_{j}+(\lambda-1) \lambda^{i_{l}} p_{l}}{\sum_{j=1}^{n} \lambda^{i_{j}} p_{j}}\right) .
\end{gathered}
$$

With the help of Theorem A, this yields that

$$
\begin{gathered}
f\left(\frac{\sum_{j=1}^{n} \lambda^{i_{j}} p_{j} x_{j}}{\sum_{j=1}^{n} \lambda^{i_{j}} p_{j}}\right) \leq \frac{1}{n+\lambda-1} \sum_{l=1}^{n}\left(\frac{\sum_{j=1}^{n} \lambda^{i_{j}} p_{j}+(\lambda-1) \lambda^{i_{l}} p_{l}}{\sum_{j=1}^{n} \lambda^{i_{j}} p_{j}}\right. \\
\left.. f\left(\frac{\sum_{j=1}^{n} \lambda^{j_{j}} p_{j} x_{j}+(\lambda-1) \lambda^{i_{l}} p_{l} x_{l}}{\sum_{j=1}^{n} \lambda^{i_{j}} p_{j}+(\lambda-1) \lambda^{i_{l}} p_{l}}\right)\right) .
\end{gathered}
$$


Consequently,

$$
\begin{gathered}
C_{k}(\lambda) \leq \frac{1}{(n+\lambda-1)^{k+1}} \sum_{\left(i_{1}, \ldots, i_{n}\right) \in S_{k}} \frac{k !}{i_{1} ! \ldots i_{n} !} \\
\cdot \sum_{l=1}^{n}\left(\left(\sum_{j=1}^{n} \lambda^{i_{j}} p_{j}+(\lambda-1) \lambda^{i_{l}} p_{l}\right) f\left(\frac{\sum_{j=1}^{n} \lambda^{i_{j}} p_{j} x_{j}+(\lambda-1) \lambda^{i_{l}} p_{l} x_{l}}{\sum_{j=1}^{n} \lambda^{i_{j}} p_{j}+(\lambda-1) \lambda^{i_{l}} p_{l}}\right)\right) .
\end{gathered}
$$

By Lemma 14, it is easy to see that the right-hand side of (13) can be written in the form

$$
\frac{1}{(n+\lambda-1)^{k+1}} \sum_{\left(i_{1}, \ldots, i_{n}\right) \in S_{k+1}} \frac{(k+1) !}{i_{1} ! \ldots i_{n} !}\left(\sum_{j=1}^{n} \lambda^{i_{j}} p_{j}\right) f\left(\frac{\sum_{j=1}^{n} \lambda^{i_{j}} p_{j} x_{j}}{\sum_{j=1}^{n} \lambda^{i_{j}} p_{j}}\right)
$$

which is just $C_{k+1}(\lambda)$.

III. Finally, we prove that

$$
C_{k}(\lambda) \leq \sum_{j=1}^{n} p_{j} f\left(x_{j}\right), \quad k \in \mathbb{N}_{+}
$$

It follows from Theorem A that

$$
\begin{aligned}
& C_{k}(\lambda) \leq \frac{1}{(n+\lambda-1)^{k}} \sum_{\left(i_{1}, \ldots, i_{n}\right) \in S_{k}}\left(\frac{k !}{i_{1} ! \ldots i_{n} !} \sum_{j=1}^{n} \lambda^{i_{j}} p_{j} f\left(x_{j}\right)\right) \\
= & \frac{1}{(n+\lambda-1)^{k}} \sum_{j=1}^{n}\left(\sum_{\left(i_{1}, \ldots, i_{n}\right) \in S_{k}} \frac{k !}{i_{1} ! \ldots i_{n} !} \lambda^{i_{j}}\right) p_{j} f\left(x_{j}\right), \quad k \in \mathbb{N}_{+} .
\end{aligned}
$$

The multinomial theorem shows that

$$
\sum_{\left(i_{1}, \ldots, i_{n}\right) \in S_{k}} \frac{k !}{i_{1} ! \ldots i_{n} !} \lambda^{i_{j}}=(n+\lambda-1)^{k}, \quad 1 \leq j \leq n,
$$

hence (15) implies (14).

The proof of Theorem 3 (a) is based on the following interesting result. The $\sigma$-algebra of Borel subsets of $\mathbb{R}^{n}$ is denoted by $\mathcal{B}^{n}$.

Lemma 15 Let $p_{1}, \ldots, p_{n}$ be a discrete distribution with $n \geq 2$, and let $\lambda>1$. Let $l \in$ $\{1, \ldots, n\}$ be fixed. $e_{l}$ denotes the vector in $\mathbb{R}^{n}$ that has 0 in all coordinate positions except the lth, where it has a 1 . Let $q_{1}, \ldots, q_{n}$ be also a discrete distribution such that $q_{j}>0(1 \leq j \leq n)$ and

$$
q_{l}>\max \left(q_{1}, \ldots q_{l-1}, q_{l+1}, \ldots, q_{n}\right) .
$$

If

$$
g:\left\{\left(t_{1}, \ldots, t_{n}\right) \in \mathbb{R}^{n} \mid t_{j}>0(1 \leq j \leq n), \quad \sum_{j=1}^{n} t_{j}=1\right\} \rightarrow \mathbb{R}
$$


is a bounded function for which

$$
\tau_{l}:=\lim _{e_{l}} g
$$

exists in $\mathbb{R}$ and $p_{l}>0$, then

$$
\lim _{k \rightarrow \infty} \sum_{\left(i_{1}, \ldots, i_{n}\right) \in S_{k}} \frac{k !}{i_{1} ! \ldots i_{n} !} q_{1}^{i_{1}} \ldots q_{n}^{i_{n}} g\left(\frac{\lambda^{i_{1}} p_{1}}{\sum_{j=1}^{n} \lambda^{i_{j}} p_{j}}, \ldots, \frac{\lambda^{i_{n}} p_{n}}{\sum_{j=1}^{n} \lambda^{i_{j}} p_{j}}\right)=\tau_{l} .
$$

Proof. To prove the result, we can obviously suppose that $l=1$.

For the sake of clarity, we shall denote the element $\left(i_{1}, \ldots, i_{n}\right)$ of $S_{k}$ by $\left(i_{1 k}, \ldots, i_{n k}\right)$ $\left(k \in \mathbb{N}_{+}\right)$.

Let $\xi_{k}:=\left(\xi_{1 k}, \ldots, \xi_{n k}\right)\left(k \in \mathbb{N}_{+}\right)$be a $\left(\mathbb{R}^{n}, \mathcal{B}^{n}\right)$-random variable on a probability space $(\Omega, \mathcal{A}, P)$ such that $\xi_{k}$ has multinomial distribution of order $k$ and with parameters $q_{1}, \ldots, q_{n}$. A fundamental theorem of the statistics (see [9], Theorem 5.4.13), which is based on the multidimensional central limit theorem and the Cochran-Fisher theorem, implies that

$$
\lim _{k \rightarrow \infty} P\left(\sum_{j=1}^{n} \frac{\left(\xi_{j k}-k q_{j}\right)^{2}}{k q_{j}}<t\right)=F_{n-1}(t), \quad t \in \mathbb{R},
$$

where $F_{n-1}$ means the distribution function of the Chi-squared distribution $\left(\chi^{2}\right.$-distribution) with $n-1$ degrees of freedom.

Choose $0<\varepsilon<1$. Since $F_{n-1}$ is continuous and strictly increasing on ]0, $\infty$ [, there exists a unique $t_{\varepsilon}>0$ such that

$$
F_{n-1}\left(t_{\varepsilon}\right)=1-\varepsilon .
$$

Define

$$
S_{k}^{1}:=\left\{\left(i_{1 k}, \ldots, i_{n k}\right) \in S_{k} \mid \sum_{j=1}^{n} k \frac{\left(\frac{i_{j k}}{k}-q_{j}\right)^{2}}{q_{j}}<t_{\varepsilon}\right\} .
$$

The definition of the set $S_{k}^{1}$ shows that

$$
\begin{gathered}
\sum_{\left(i_{1 k k}, \ldots, i_{n k}\right) \in S_{k}^{1}} \frac{k !}{i_{1 k} ! \ldots i_{n k} !} q_{1}^{i_{1 k}} \ldots q_{n}^{i_{n k}}=P\left(\left(\xi_{1 k}, \ldots, \xi_{n k}\right) \in S_{k}^{1}\right) \\
=P\left(\sum_{j=1}^{n} k \frac{\left(\frac{\xi_{j k}}{k}-q_{j}\right)^{2}}{q_{j}}<t_{\varepsilon}\right)=P\left(\sum_{j=1}^{n} \frac{\left(\xi_{j k}-k q_{j}\right)^{2}}{k q_{j}}<t_{\varepsilon}\right) \\
=F_{n-1}\left(t_{\varepsilon}\right)+\left(P\left(\sum_{j=1}^{n} \frac{\left(\xi_{j k}-k q_{j}\right)^{2}}{k q_{j}}<t_{\varepsilon}\right)-F_{n-1}\left(t_{\varepsilon}\right)\right) \\
=1-\varepsilon+\delta_{\varepsilon}(k), \quad k \in \mathbb{N}_{+}
\end{gathered}
$$


where by (18)

$$
\lim _{k \rightarrow \infty} \delta_{\varepsilon}(k)=0 .
$$

For $j=1, \ldots, n$ construct the sequences $\left(I_{k}^{j}\right)_{k \geq 1}$ by

$$
I_{k}^{j}:=i_{j k^{\prime}}^{*} \text { if }\left|\frac{i_{j k}^{*}}{k}-q_{j}\right|=\max \left\{\left|\frac{i_{j k}}{k}-q_{j}\right| \mid\left(i_{1 k}, \ldots, i_{n k}\right) \in S_{k}^{1}\right\}, \quad k \in \mathbb{N}_{+} .
$$

We claim that

$$
\lim _{k \rightarrow \infty} \frac{I_{k}^{j}}{k}=q_{j}, \quad 1 \leq j \leq n .
$$

Fix $1 \leq j \leq n$. If (23) is false, then (22) yields that we can find a positive number $\rho$, a strictly increasing sequence $\left(k_{u}\right)_{u \geq 1}$ and points

$$
\left(i_{1 k_{u^{\prime}}}, \ldots, i_{n k_{u}}\right) \in S_{k_{u^{\prime}}}^{1}, \quad u \in \mathbb{N}_{+}
$$

such that

$$
\left|\frac{i_{j k_{u}}}{k_{u}}-q_{j}\right| \geq \rho, \quad u \in \mathbb{N}_{+}
$$

and therefore,

$$
k_{u} \frac{\left(\frac{i_{j k_{u}}}{k_{u}}-q_{j}\right)^{2}}{q_{j}} \geq k_{u} \frac{\rho^{2}}{q_{j}} \rightarrow \infty \text { as } u \rightarrow \infty,
$$

contrary to (24).

Let

$$
q:=\max \left(q_{2}, \ldots, q_{n}\right) .
$$

It follows from (16) that

$$
\gamma:=\frac{1}{3}\left(q_{1}-q\right)>0
$$

By (22) and (23), we can find an integer $k_{\gamma}$ such that for each $k>k_{\gamma}$

$$
\left|\frac{i_{j k}}{k}-q_{j}\right| \leq\left|\frac{I_{k}^{j}}{k}-q_{j}\right|<\gamma, \quad\left(i_{1 k}, \ldots, i_{n k}\right) \in S_{k^{\prime}}^{1} \quad 1 \leq j \leq n .
$$

Thus, for every $k>k_{\gamma}$

$$
\frac{i_{1 k}}{k}>q_{1}-\gamma \text { and } \frac{i_{j k}}{k}<q_{j}+\gamma, \quad 2 \leq j \leq n, \quad\left(i_{1 k}, \ldots, i_{n k}\right) \in S_{k}^{1},
$$

and hence, we get from (25) that

$$
i_{1 k}-i_{j k}>k \gamma \quad 2 \leq j \leq n, \quad\left(i_{1 k}, \ldots, i_{n k}\right) \in S_{k^{\prime}}^{1} \quad k>k_{\gamma} .
$$

We can see that

$$
i_{1 k}-i_{j k} \rightarrow \infty \text { as } k \rightarrow \infty, \quad 2 \leq j \leq n, \quad\left(i_{1 k}, \ldots, i_{n k}\right) \in S_{k}^{1} .
$$


Now, set $S_{k}^{2}:=S_{k} \backslash S_{k}^{1}\left(k \in \mathbb{N}_{+}\right)$, and consider the sequences

$$
a_{k}^{1}:=\sum_{\left(i_{1 k,}, \ldots, i_{n k}\right) \in S_{k}^{1}} \frac{k !}{i_{1 k} ! \ldots i_{n k} !} q_{1}^{i_{1 k}} \ldots q_{n}^{i_{n k} g}\left(\frac{\lambda^{i_{1 k}} p_{1}}{\sum_{j=1}^{n} \lambda^{i_{j k}} p_{j}}, \ldots, \frac{\lambda^{i_{n k}} p_{n}}{\sum_{j=1}^{n} \lambda^{i_{k k}} p_{j}}\right),
$$

and

$$
a_{k}^{2}:=\sum_{\left(i_{1 k,}, \ldots, i_{n k}\right) \in S_{k}^{2}} \frac{k !}{i_{1 k} ! \ldots i_{n k} !} q_{1}^{i_{1 k}} \ldots q_{n}^{i_{n k} g}\left(\frac{\lambda^{i_{1 k}} p_{1}}{\sum_{j=1}^{n} \lambda^{i_{j k}} p_{j}}, \ldots, \frac{\lambda^{i_{n k}} p_{n}}{\sum_{j=1}^{n} \lambda^{i_{j k}} p_{j}}\right),
$$

where $k \in \mathbb{N}_{+}$. The sum of these sequences is just the studied sequence in (17). Since $p_{1}>0$, we obtain from (27) that

$$
\lim _{k \rightarrow \infty} \frac{\lambda^{i_{1 k}} p_{1}}{\sum_{j=1}^{n} \lambda^{i_{j k}} p_{j}}=1, \quad\left(i_{1 k}, \ldots, i_{n k}\right) \in S_{k}^{1}
$$

and

$$
\lim _{k \rightarrow \infty} \frac{\lambda^{i_{k}} p_{1}}{\sum_{j=1}^{n} \lambda^{i_{j k}} p_{j}}=0, \quad 2 \leq l \leq n, \quad\left(i_{1 k}, \ldots, i_{n k}\right) \in S_{k}^{1} .
$$

According to (26), the convergence is uniform for all the possible sequences in (28) and (29); hence, for every $\varepsilon_{1}>0$, we can find an integer $k_{\varepsilon_{1}}>k_{\gamma}$ that for all $k>k_{\varepsilon_{1}}$

$$
\tau_{1}-\varepsilon_{1}<g\left(\frac{\lambda^{i_{1 k}} p_{1}}{\sum_{j=1}^{n} \lambda^{i_{k k}} p_{j}}, \ldots, \frac{\lambda^{i_{n k}} p_{n}}{\sum_{j=1}^{n} \lambda^{i_{k k}} p_{j}}\right)<\tau_{1}+\varepsilon_{1}, \quad\left(i_{1 k}, \ldots, i_{n k}\right) \in S_{k}^{1} .
$$

Bringing in (19-20), we find that

$$
P\left(\left(\xi_{1 k}, \ldots, \xi_{n k}\right) \in S_{k}^{2}\right)=\varepsilon-\delta_{\varepsilon}(k), \quad k \in \mathbb{N}_{+},
$$

and therefore, thanks to (19-20), (30) and the boundedness of $g(|g| \leq m)$

$$
\begin{aligned}
& \left(1-\varepsilon+\delta_{\varepsilon}(k)\right)\left(\tau_{1}-\varepsilon_{1}\right)-\left(\varepsilon-\delta_{\varepsilon}(k)\right) m \leq a_{k}^{1}+a_{k}^{2} \\
\leq & \left(1-\varepsilon+\delta_{\varepsilon}(k)\right)\left(\tau_{1}+\varepsilon_{1}\right)+\left(\varepsilon-\delta_{\varepsilon}(k)\right) m, \quad k>k_{\varepsilon_{1}} .
\end{aligned}
$$

Consequently, by (21)

$$
\begin{gathered}
(1-\varepsilon)\left(\tau_{1}-\varepsilon_{1}\right)-\varepsilon m \leq \liminf _{k \rightarrow \infty}\left(a_{k}^{1}+a_{k}^{2}\right) \leq \limsup _{k \rightarrow \infty}\left(a_{k}^{1}+a_{k}^{2}\right) \\
\leq(1-\varepsilon)\left(\tau_{1}+\varepsilon_{1}\right)+\varepsilon m,
\end{gathered}
$$

and this proves the convergence claim (17).

The proof is now complete.

The proof of Theorem 3 . 
Proof. (a) We have only to observe that for every fixed $1 \leq l \leq n$

$$
\lim _{k \rightarrow \infty} \frac{1}{(n+\lambda-1)^{k}} \sum_{\left(i_{1}, \ldots, i_{n}\right) \in S_{k}} \frac{k !}{i_{1} ! \ldots i_{n} !} \lambda^{i_{l}} p_{l} f\left(\frac{\sum_{j=1}^{n} \lambda^{i_{j}} p_{j} x_{j}}{\sum_{j=1}^{n} \lambda^{i_{j}} p_{j}}\right)=p_{l} f\left(x_{l}\right) .
$$

The case $p_{l}=0$ is trivial.

To prove the case $p_{l}>0$, define the function

$$
g:\left\{\left(t_{1}, \ldots, t_{n}\right) \in \mathbb{R}^{n} \mid t_{j}>0(1 \leq j \leq n), \quad \sum_{j=1}^{n} t_{j}=1\right\} \rightarrow \mathbb{R}
$$

by

$$
g\left(t_{1}, \ldots, t_{n}\right):=f\left(\sum_{j=1}^{n} t_{j} x_{j}\right) .
$$

Consequently, the limit in (31) can be written in the form

$$
\begin{gathered}
\lim _{k \rightarrow \infty} p_{l} \sum_{\left(i_{1}, \ldots, i_{n}\right) \in S_{k}} \frac{k !}{i_{1} ! \ldots i_{n} !}\left(\frac{1}{n+\lambda-1}\right)^{i_{1}} \cdots\left(\frac{1}{n+\lambda-1}\right)^{i_{l-1}}\left(\frac{\lambda}{n+\lambda-1}\right)^{i_{l}} \\
.\left(\frac{1}{n+\lambda-1}\right)^{i_{l+1}} \cdots\left(\frac{1}{n+\lambda-1}\right)^{i_{n}} g\left(\frac{\lambda^{i_{1}} p_{1}}{\sum_{j=1}^{n} \lambda^{i_{j}} p_{j}}, \ldots, \frac{\lambda^{i_{n}} p_{n}}{\sum_{j=1}^{n} \lambda^{i_{j}} p_{j}}\right) .
\end{gathered}
$$

Now, we can apply Lemma 15 with

$$
q_{j}=\frac{1}{n+\lambda-1}, \quad 1 \leq j \leq n, \quad j \neq l, \text { and } q_{l}=\frac{\lambda}{n+\lambda-1}
$$

and

$$
\lim _{e_{l}} g=f\left(x_{l}\right), \quad 1 \leq l \leq n .
$$

(b) Elementary considerations show this part of the theorem.

The proof is complete.

The proof of Theorem 5.

Proof. Theorem A confirms that $f$ is bounded on the set

$$
G:=\left\{\sum_{j=1}^{n} t_{j} x_{j} \in C \mid t_{j} \geq 0 \quad(1 \leq j \leq n), \quad \sum_{j=1}^{n} t_{j}=1\right\},
$$

where $t_{j}(1 \leq j \leq n)$ is also rational if $f$ is mid-convex.

It is elementary that for every $\left(i_{1}, \ldots, i_{n}\right) \in S_{k}$

$$
\lim _{\lambda \rightarrow \infty} \frac{\lambda^{i_{l}}}{(n+\lambda-1)^{k}}=\left\{\begin{array}{l}
1, \text { if } i_{l}=k \\
0, \text { if } i_{l}<k^{\prime}
\end{array} \quad 1 \leq l \leq n .\right.
$$


By the definition of the set $S_{k},(0, \ldots, 0, k, 0, \ldots, 0)$ (the vector has $0 \mathrm{~s}$ in all coordinate positions except the $l$ th) is the only element of $S_{k}$ for which $i_{l}=k(1 \leq l \leq n)$. By using the boundedness of $f$ on $G$, the previous assumptions imply the result, bringing the proof to an end.

\section{Acknowledgements}

This study was supported by the Hungarian National Foundations for Scientific Research Grant No. K73274.

\section{Competing interests}

The author declares that he has no competing interests.

Received: 8 March 2011 Accepted: 25 July 2011 Published: 25 July 2011

\section{References}

1. Hardy, GH, Littlewood, JE, Pólya, G: Inequalities. Cambridge Mathematical Library Series. Cambridge University Press (1967)

2. Pečarić, JE, Volenec, V: Interpolation of the Jensen inequality with some applications, Österreích. Akad Wiss Math-Natur KI Sitzungsber II. 197, 463-467 (1988)

3. Pečarić, JE, Svrtan, D: Unified approach to refinements of Jensen's inequalities. Math Inequal Appl. 5, 45-47 (2002)

4. Horváth, L, Pečarić, JE: A refinement of the discrete Jensen's inequality. Math Inequal Appl. (to appear)

5. Horváth, L: A method to refine the discrete Jensen's inequality for convex and mid-convex functions. doi:10.1016/j. mcm.2011.05.060

6. Horváth, L: Inequalities corresponding to the classical Jensen's inequality. J Math Inequal. 3, 189-200 (2009)

7. Xiao, Z-G, Srivastava, HM, Zhang, Z-H: Further refinements of the Jensen inequalities based upon samples with repetitions. Math Comput Mod. 51, 592-600 (2010)

8. Mitrinović, DS, Pečarić, JE, Fink, AM: Classical and New Inequalities in Analysis, vol. 61 of Mathematics and Its Applications. Kluwer Academic Publishers, Dordrecht (1993)

9. Dacunha-Castello, D, Duflo, M: Probability and Statistics I. Springer-Verlag, New York (1986)

doi:10.1186/1029-242X-2011-26

Cite this article as: Horváth: A parameter-dependent refinement of the discrete Jensen's inequality for convex and mid-convex functions. Journal of Inequalities and Applications 2011 2011:26.

\section{Submit your manuscript to a SpringerOpen ${ }^{\circ}$ journal and benefit from:}

Convenient online submission

- Rigorous peer review

- Immediate publication on acceptance

- Open access: articles freely available online

- High visibility within the field

- Retaining the copyright to your article

Submit your next manuscript at $>$ springeropen.com 